\title{
Propofol adsorption at the air/water interface: a combined vibrational sum frequency spectroscopy, nuclear magnetic resonance and neutron reflectometry study
} DOI:

10.1039/C8SM01677A

\section{Document Version \\ Accepted author manuscript}

Link to publication record in Manchester Research Explorer

Citation for published version (APA):

Niga, P., Hansson-mille, P. M., Swerin, A., Claesson, P. M., Schoelkopf, J., Gane, P. A. C., Dai, J., Furó, I., Campbell, R. A., \& Johnson, C. M. (2019). Propofol adsorption at the air/water interface: a combined vibrational sum frequency spectroscopy, nuclear magnetic resonance and neutron reflectometry study. Soft Matter, 15(1), 3846. https://doi.org/10.1039/C8SM01677A

\section{Published in:}

Soft Matter

\section{Citing this paper}

Please note that where the full-text provided on Manchester Research Explorer is the Author Accepted Manuscript or Proof version this may differ from the final Published version. If citing, it is advised that you check and use the publisher's definitive version.

\section{General rights}

Copyright and moral rights for the publications made accessible in the Research Explorer are retained by the authors and/or other copyright owners and it is a condition of accessing publications that users recognise and abide by the legal requirements associated with these rights.

\section{Takedown policy}

If you believe that this document breaches copyright please refer to the University of Manchester's Takedown Procedures [http://man.ac.uk/04Y6Bo] or contact uml.scholarlycommunications@manchester.ac.uk providing relevant details, so we can investigate your claim.

\section{OPEN ACCESS}




\section{ARTICLE}

Received 00th January 20xx Accepted 00th January 20xx DOI: $10.1039 / \mathrm{x} 0 \mathrm{xx} 00000 \mathrm{x}$ www.rsc.org/

\section{Propofol adsorption at the air/water interface: a combined vibrational sum frequency spectroscopy, nuclear magnetic resonance and neutron reflectometry study.}

Petru Niga ${ }^{a}$, Petra M. Hansson-Mille ${ }^{a}$, Agne Swerin ${ }^{a, b}$, Per M. Claesson ${ }^{a, b}$, Joachim Schoelkopf ${ }^{c}$, Patrick A. C. Gane ${ }^{c, d}$, Jing Dai ${ }^{e}$, István Furó ${ }^{e}$, Richard A. Campbell ${ }^{f, g}$ and C. Magnus Johnson ${ }^{\text {* }}$

Propofol is an amphiphilic small molecule that strongly influences the function of cell membranes, yet data regarding interfacial properties of propofol remain scarce. Here we consider propofol adsorption at the air/water interface as elucidated by means of vibrational sum frequency spectroscopy (VSFS), neutron reflectometry (NR), and surface tensiometry. VSFS data show that propofol adsorbed at the air/water interface interacts with water strongly in terms of hydrogen bonding and weakly in the proximity of the hydrocarbon parts of the molecule. In the concentration range studied there is almost no change in the orientation adopted at the interface. Data from NR show that propofol forms a dense monolayer with a thickness of $8.4 \AA$ and a limiting area per molecule of $40 \AA^{2}$, close to the value extracted from surface tensiometry. The possibility that islands or multilayers of propofol form at the air/water interface is therefore excluded as long as the solubility limit is not exceeded. Additionally, nuclear magnetic resonance (NMR) measurements of the ${ }^{1} \mathrm{H}$ nuclear magnetic resonance chemical shifts demonstrate that propofol does not form dimers or multimers in bulk water up to the solubility limit.

\section{Introduction}

Alcohol/water mixtures are widely used as industrial solvents and chemical reagents, and the need of a better understanding of the interfacial behaviour of aqueous alcohol solutions at different interfaces has led to increasing interest in fundamental research. ${ }^{1,2}$ The behaviour of alcohols at

RISE Research Institutes of Sweden - Chemistry, Materials and Surfaces, Box 5607, SE-114 86 Stockholm, Sweden.

b. KTH Royal Institute of Technology, Department of Chemistry, Division of Surface and Corrosion Science, SE - 10044 Stockholm, Sweden.

c. Omya International AG, Baslerstrasse $42, \mathrm{CH}-4665$ Oftringen, Switzerland.

d. Aalto University, School of Chemical Technology, Department of Bioproducts and Biosystems, FI-00076 Aalto, Helsinki, Finland.

e. KTH Royal Institute of Technology, Department of Chemistry, Division of Applied Physical Chemistry, SE - 10044 Stockholm, Sweden.

f. Institut Laue-Langevin, 71 Avenue des Martyrs, CS20156, 38042 Grenoble Cedex 9, France

g. Division of Pharmacy and Optometry, University of Manchester, Manchester M13 9PT, UK solid interfaces plays a key role in applications like cleaning, etching, and electrochemical reactions. ${ }^{3,4}$ Let alone the industrial applications, the properties of alcohols at buried interfaces have important implications in cell membrane function. The functioning of intrinsic membrane proteins can be altered by introduction of short chain alcohols (e.g. propofol) at the membrane surface. ${ }^{5,6}$ As a result, propofol has, for example, been shown to induce a significant change in membrane permeability. ${ }^{7}$ This is potentially important since low molecular weight anaesthetics are used daily in hospitals, but yet the molecular mechanism of general anaesthesia remains to some extent ambiguous. ${ }^{8}$

In spite of the importance of interfacial properties of alcohol/water mixtures in a wide variety of applications ${ }^{9,10}$ the study of such interfaces remain complex, mainly due to their dynamic nature and the lack of surface-specific experimental techniques. The development of nonlinear optical methods has contributed to overcoming some of these problems and facilitated better molecular understanding of such 
interfaces. Specifically, vibrational sum frequency spectroscopy (VSFS) has proven to be a powerful technique for studying a wide variety of aqueous interfaces $^{11}$ due to its capability of yielding surfacespecific information at a molecular level.

Different types of alcohols have been studied using VSFS with respect to the stretching vibrations of the hydrocarbon chains, ${ }^{11-14}$ the fingerprint region ${ }^{15}$ and the water of hydration. ${ }^{16-20}$ It was found that for a series of increasing chain length alcohols (C1-C8), at the neat air/alcohol interface, the tails point out into the gas phase due to the amphiphilic character of alcohols, and that the presence of gauche defects is chain length dependent. At the same time a very well ordered interfacial hydrogen bonding network was detected. ${ }^{21}$ Short chain alcohols (C1-C3) do not affect the interfacial hydrogen bonding structure and orientation to a large extent, while long chain alcohols (C5-C12) make the interfacial water more strongly hydrogen bonded and reversely oriented. ${ }^{20}$ In mixtures with water, glycerol was found to partition to the interface with air, whereby disturbing the topmost water layers. ${ }^{18}$ At a hydrophobic solid surface, methanol adsorbed from its mixture with water to the interface with the $\mathrm{C}-\mathrm{O}$ bond aligned to the surface normal while the water band at $3200 \mathrm{~cm}^{-1}$ weakened in strength with increasing methanol concentration. ${ }^{16}$

The knowledge of small hydrophobic but weakly amphiphilic drugs at the interface is rather limited compared with the more amphiphilic molecules. Amphiphilic molecules such as surfactants $^{22}$, proteins ${ }^{23}$, block co-polymers ${ }^{24}$ and peptides $^{25}$ have been the subject of extensive investigations. By contrast there is a lack of body of work on the surface properties of small hydrophobic drugs. For example, for ibuprofen there has been a study on its interactions with lipid bilayers ${ }^{26}$ and cholesterol $^{27}$ but to our knowledge not on its adsorption properties at the air/water interface. A recent study looked at the interactions of testosterone enanthate with surfactants at the air/water interface $^{28}$, but again to our knowledge the surface properties of the drug alone have not been examined explicitly. Further, for propofol the present authors have conducted a study on its interactions with phospholipids monolayers ${ }^{29}$ but an analogous study of its adsorption properties at the air/water interface is missing. To address this shortcoming, we have decided to study its adsorption at the air/water interface.

Propofol is a small alcohol that contains both hydrophobic and hydrophilic parts and has interesting biochemical properties, e.g., it is used commonly in general anaesthesia to induce a state of reduced consciousness in patients during medical procedures. ${ }^{30-32}$ Similar in structure to propofol, phenol has been briefly characterised by VSFS at the air/water interface, ${ }^{15}$ by focusing on only the carbonoxygen stretching region of the spectrum. It was found that phenol is present at the interface at both low and high $\mathrm{pH}$, and at high $\mathrm{pH}$ there are both phenol and phenolate ions. Surprisingly however, according to our knowledge, there are no detailed studies of propofol at the air/water or water/hydrophobic interface despite its fundamental interest as a small amphiphilic drug with important biomedical applications.

In the present work we evaluate the adsorption isotherm from surface tension data, and the adsorbed amount is also determined by neutron reflectivity (NR) measurements. NR and VSFS data are then combined to gain structural information on the adsorbed layer and its interaction with water. We also report a bulk study of the chemical shifts of hydrogen nuclei over the entire solubility range of propofol in water in order to detect possible multimeric structures. The results represent a necessary first step in resolving the driving forces for the interactions of propofol with interfaces, and they set the stage for future studies involving its interactions in more complex systems (including supported lipid bilayers ${ }^{33}$, ${ }^{34}$ and membrane proteins ${ }^{35,36}$ ) that are closer in nature to those in practical applications of the drug.

\section{Materials and Methods}

\section{Materials}

Propofol (European Pharmacopoeia) with purity higher than $99 \%$ was bought from Sigma Aldrich. Its chemical structure is presented in Figure 1, where also the NMR chemical shifts are listed. A Millipore Milli-Q Plus system was used as water supply, providing purified water with a resistivity of $18.2 \mathrm{M} \Omega \mathrm{cm}$.

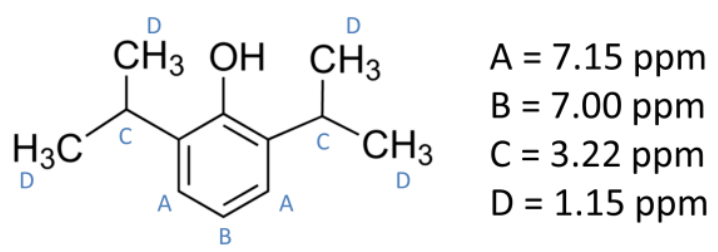

Figure 1. The chemical structure of propofol together with the ${ }^{1} \mathrm{H}$ NMR peak assignments. 


\section{Nuclear Magnetic Resonance}

${ }^{1} \mathrm{H}$ NMR spectra of propofol dissolved in $\mathrm{D}_{2} \mathrm{O}$ were obtained for a concentration series on a Bruker Advance III $500 \mathrm{MHz}$ spectrometer using a $5 \mathrm{~mm}$ Bruker DIFF 30 probe. All experiments were performed at $293 \mathrm{~K}$ and the chemical shifts of the different propofol peaks were recorded; as spectral reference, we used the ${ }^{1} \mathrm{HDO}$ signal (set to $4.75 \mathrm{ppm}$ ).

\section{Surface Tensiometry}

A Du Noüy ring instrument (Krüss) was used to determine the surface tension of aqueous propofol solutions. An independent solution was prepared for each concentration. To estimate the surface excess from the surface tension measurements, we used the Gibbs equation assuming only a single adsorbing neutral species, as shown in the equation below.

$\Gamma_{\mathrm{ST}}=-\frac{1}{\mathrm{R} T} \frac{\mathrm{d} \gamma}{\mathrm{d} \ln c}$

where $\Gamma_{\mathrm{ST}}$ is the surface excess as measured by the surface tension method, $\gamma$ is the surface tension, $c$ is the bulk concentration, $R$ is the gas constant, and $T$ is the absolute temperature. Since the NMR data reported below do not show any evidence for dimerisation, eq. 1 , where the concentration is used instead of the activity, is judged to be a good approximation up to the solubility limit.

The solutions were prepared by dropwise adding liquid propofol to water, then gently shaking and hand warming for a few minutes until the propofol was dissolved. At least $10 \mathrm{~min}$ was allowed before the measurements were recorded. For higher concentrations it took longer time for propofol to completely dissolve.

\section{Neutron Reflectometry (NR)}

NR measurements were performed using the time-of-flight reflectometer FIGARO at the Institut Laue-Langevin (Grenoble, France) ${ }^{37}$. The neutron reflectometry determines the ratio of the number of neutrons in the specular reflection to those in the incident beam with respect to the momentum transfer, $\mathrm{q}_{\mathrm{z}}$,

$q_{\mathrm{z}}=\frac{4 \pi \sin \theta}{\lambda}$

where $\lambda$ is the neutron wavelength and $\theta$ is the angle of incidence ${ }^{38}$. Data were recorded with $7 \% \delta \lambda / \lambda$ using a frame overlap mirror of $16 \AA$. The surface excess of propofol at the air/water interface at six different bulk concentrations was recorded at $\theta=$ $0.623^{\circ}$ for $15 \mathrm{~min}$, where the solvent was air contrast matched water (ACMW), which is $8.1 \%$ by volume $D_{2} \mathrm{O}$

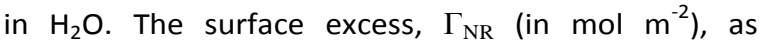
determined by neutron reflection was calculated using:

$\Gamma_{\mathrm{NR}}=\frac{\rho \cdot d}{\mathrm{~N}_{\mathrm{A}} \cdot \sum b_{i}}$

where $\rho$ is the scattering length density of propofol, $d$ the fitted layer thickness, $\mathrm{N}_{\mathrm{A}}$ is Avogadro's number and $\sum b_{i}$ is the scattering length of propofol. Note that the scattering length density of propofol was calculated as $6.2 \times 10^{-7} \AA^{-2}$ according to its molecular volume of $285 \AA^{3}$ (which is calculated from bulk density).

Two different data acquisition approaches were used to determine: (1) the adsorption isotherm and (2) the interfacial structure of the adsorbed molecules at the air/water interface, following the methodology adopted in ref. $^{39}$ In the first case, measurements were made only in ACMW, and the data were reduced only over $4.5-12 \AA$ to restrict the $q_{z}$-range to $0.01-0.03 \AA^{-1}$. This approach reduces the sensitivity of the analysis to details of the structure at the interface. ${ }^{40}$ Note that the surface excess of propofol could be resolved accurately even though it was used in its normal, non-deuterated form thanks to the high flux at low- $q_{z}$ of FIGARO. The interfacial roughness values were fixed to $3.1 \AA$, consistent with the outcome of the structural analysis (note that for this low- $q_{z}$ analysis even neglect of the roughness values resulted in a change in surface excess of $<0.5$ $\%)$. The background was not subtracted from these data in this analysis, but its accurate determination is essential to the precise quantification of the scattering excess: its value was iterated from the lowest value possible, where a layer of zero surface excess could be fitted to a measurement of pure ACMW. ${ }^{41}$

In the second case, for the structural analysis, data were recorded at a propofol concentration of $0.89 \mathrm{mM}$ over a broader $q_{\mathrm{z}}$-range with measurements at $\theta=0.623$ o and $\theta=3.78$ o both in ACMW and D2O. The background was subtracted from these data with the use of the $2 D$ detector on the instrument. A singlelayer structural model adequately described the data. In this case the fitting parameters were the thickness of the layer and its volume fraction. The roughness values of the air/propofol and propofol/water interfaces were constrained to be equal to each other 
and consistent with capillary wave theory, following an approach described recently. ${ }^{42}$ The data analysis was performed exclusively using Motofit. ${ }^{43}$

\section{Vibrational Sum Frequency Spectroscopy}

The experimental VSF spectrometer has been described previously ${ }^{44}$. A $1064 \mathrm{~nm}$ laser beam generated from a Nd:YAG laser system (PL-2251A-20, Ekspla) is used to pump the optical parameter generator/optical parameter amplifier OPG/OPA (LaserVision) system, which produces a fixed visible beam $(532 \mathrm{~nm})$ and a tuneable IR beam (1000-4000 $\mathrm{cm}-1)$. These two laser beams overlap at the sample surface with incident angles of $55^{\circ}$ and $63^{\circ}$ from the surface normal for the visible and IR beams, respectively. The VSF beam is collected and filtered both optically and spatially before being sent to a photomultiplier tube (Jobin Yvon). The signal is integrated in a boxcar and finally processed by a computer program.

The theory behind VSFS is well described in the literature. ${ }^{45-49}$ It is a nonlinear optical technique, which is able to provide surface specific information such as the nature and orientation of the species present at an interface. The intensity of the collected beam is proportional to the intensities of the incoming visible and infrared beams and the square of the effective second order nonlinear susceptibility $\chi^{(2)}$ eff . $\chi_{\text {eff }}^{(2)}$ is in turn proportional to the number density multiplied by the orientationally averaged molecular hyperpolarisability $\left\langle\beta^{(2)}>\right.$ of the probed species. We note that in order for a molecule or part of a molecule to be VSF active it has to be both IR and Raman active, according to Eq. (4):

$\beta_{\alpha \beta \gamma}^{(2)}=\frac{\alpha_{\alpha \beta} \mu_{\gamma}}{\omega_{n}-\omega_{\mathrm{IR}}-i \Gamma_{n}}$

where $\alpha, \beta$, and $\gamma$ are the molecular coordinates, $\alpha_{\alpha \beta}$ is the Raman polarisability tensor, $\mu_{\gamma}$ the transition IR dipole moment, $\omega_{n}$ the peak position frequency, $\omega_{\mathrm{IR}}$ the irradiating infrared frequency, $\Gamma_{n}$ the damping constant of the $n^{\text {th }}$ resonant mode and $i$ the imaginary unit. The transformation of the molecular hyperpolarisability coordinates into laboratory coordinates is done using an Euler transformation matrix. ${ }^{50}$ The obtained VSF spectra were fitted using a Lorentzian line profile using an IgorPro (WaveMetrics) program:

$I_{\mathrm{VSF}}=\left|A_{\mathrm{NR}}+\sum_{n} \frac{A_{n}}{\omega_{n}-\omega_{\mathrm{IR}}-i \Gamma_{n}}\right|^{2}$ where $I_{\mathrm{VSF}}$ is the recorded VSF signal, $A_{\mathrm{NR}}$ is the nonresonant amplitude of the VSF signal and $A_{n}$ is the amplitude (oscillator strength) of the $n^{\text {th }}$ resonant mode.

Orientational information about the probed molecular species is given by elements of the second order nonlinear susceptibility tensor, specifically by using different polarisation combinations. In this study we have used SSP, PPP, and SPS, where $P$ refers to light polarised parallel to the plane of incidence and $S$ refers to light polarised perpendicular to the plane of incidence. The first letter corresponds to the polarisation of the sum frequency beam, the second to that of the visible beam, and the third to the infrared beam.

\section{Results and discussion}

\section{Propofol in Aqueous Bulk Solution}

The ${ }^{1} H$ NMR spectrum of propofol has been published before, ${ }^{51,52}$ and therefore it is not shown here. As is clear from Figure 2, the chemical shifts of the hydrogen atoms in propofol remain constant in the concentration range $0.02-0.89 \mathrm{mM}$. Since propofol contains an aromatic moiety with large resulting "ring current" effects, any aggregation should have a large effect on the observed shifts. ${ }^{53,54}$

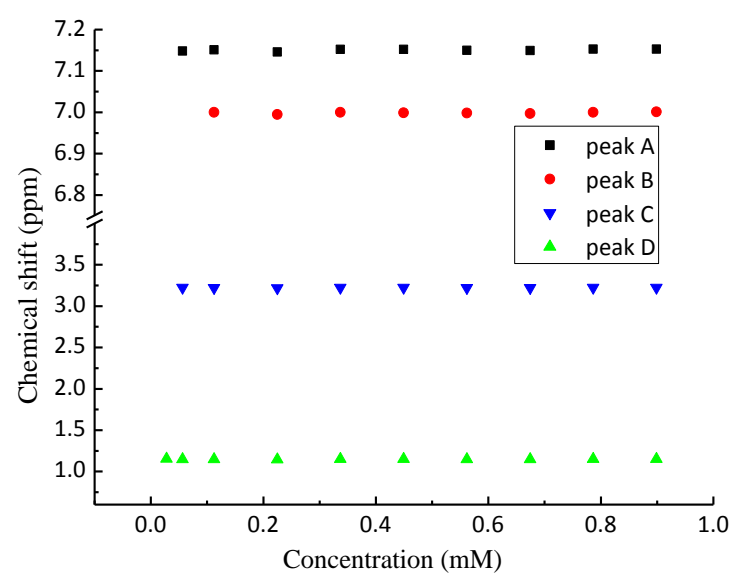

Figure 2. The chemical shifts (see Figure 1 for peak assignment) recorded in solution of propofol at different concentrations between $0.02 \mathrm{mM}$ and 0.89 mM. A - aromatic $\mathrm{H} 3$ and $\mathrm{H} 5, \mathrm{~B}-$ aromatic $\mathrm{H} 4, \mathrm{C}-\mathrm{CH}(\mathrm{CH} 3) 2$, and $\mathrm{D}-\mathrm{CH} 3$.

The absence of such effects points to the absence of any significant aggregation/dimerisation phenomena. This is in contradiction to what has been inferred in gas-phase where "nano-micelles" containing several multimers of propofol were found. $^{55}$ In addition, the NMR intensity was proportional to the concentration in the whole range 
which also excludes the presence of any large aggregates (for which the NMR signal could possibly be lost).

\section{Propofol Adsorption Isotherm}

In order to gain insight into the adsorption process of propofol at the air/water interface we have combined information from surface tensiometry, NR and VSFS.
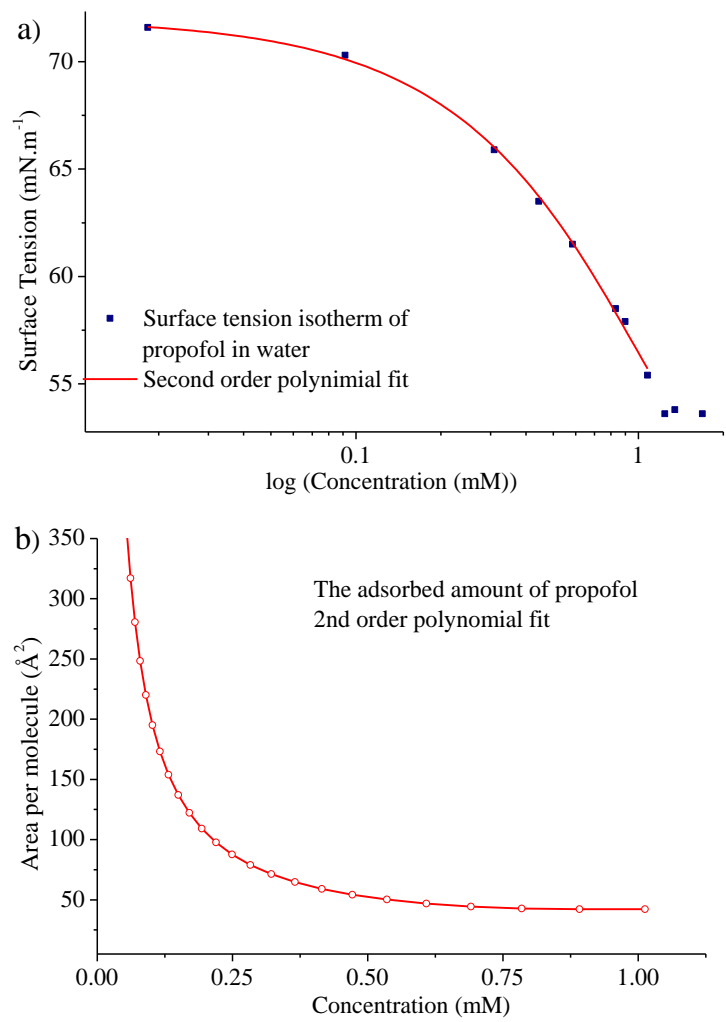

Figure 3. a) Surface tension isotherm of propofol and a second order polynomial fit to the data (line), and b) area per molecule calculated using the second order polynomial fit and eq. 1.

Figure 3a shows the surface tension isotherm of aqueous propofol solutions. The decrease in the surface tension is due to adsorption of propofol at the air/water interface. The surface tension drops smoothly with concentration up to a concentration of about $1 \mathrm{mM}$, above which it remains constant. The limiting area per molecule is about $42 \AA^{2}$, as seen in Figure $3 \mathrm{~b}$. A discussion regarding the structure of the adsorbed propofol layer will follow in the light of the NR results presented next. Details on area per molecules calculation are given in Supporting Information.

The surface excess was measured directly at six different bulk concentrations at low $q_{z}$ values in
ACMW, as shown in Figure 4a. Also shown is the measurement of pure ACMW used to determine the background level. The resulting adsorption isotherm is shown in Figure 4b, where it is compared to the surface excess obtained from surface tension measurements. We regard the agreement as satisfactory, particularly at high propofol concentrations, considering the different evaluation methods.
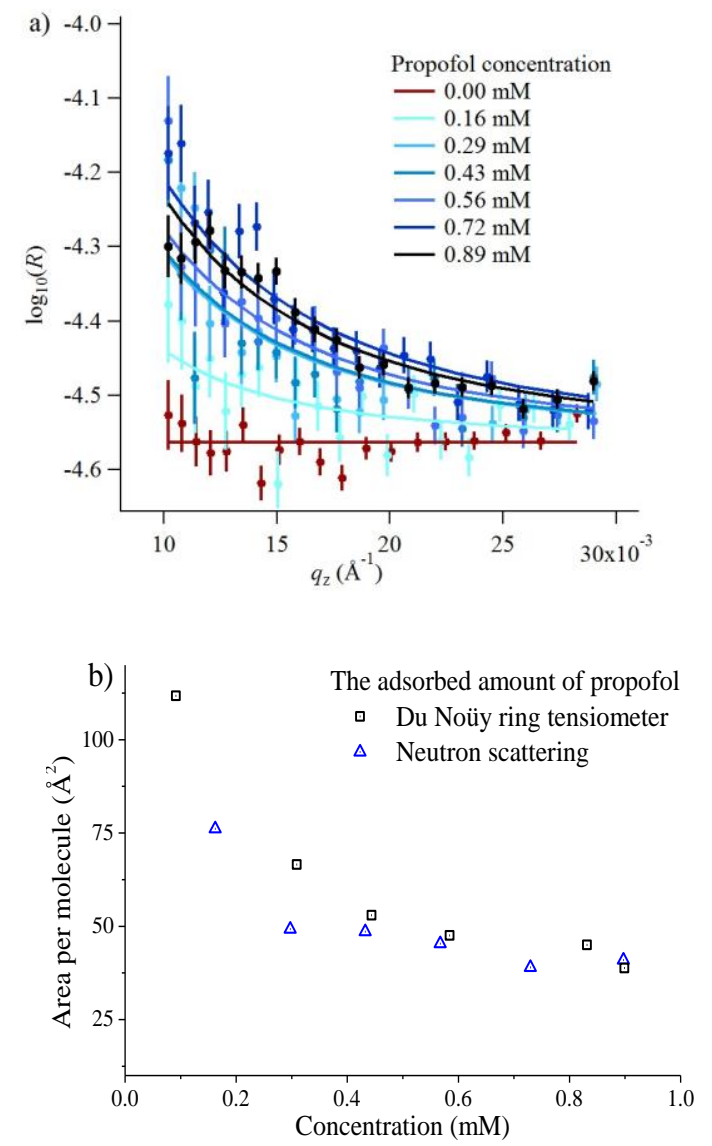

Figure 4. a) Neutron reflectometry data, $R$, and model fits for propofol solutions at the air/water interface, where the six bulk concentrations indicated are drawn progressively darker in colour; the pure ACMW background data are shown in red, and model fits are shown as lines; b) a comparison of the area per molecule obtained from surface tension measurements and NR.

\section{Propofol Interfacial Organisation}

In Figure 5, the NR data and model fits for a $0.89 \mathrm{mM}$ propofol solution at the air/water interface are presented. The inter-layer roughness values were again constrained to the capillary wave value of $3.1 \AA$, the residual background was fitted to $2 \times 10^{-7}$ (a.u.), and the thickness of the propofol monolayer and its volume fraction were fitted. The fit result using a generic algorithm converged to a dense propofol layer 
of $8.4 \AA$ thicknesses with a volume fraction of 0.86 . These demonstrate that propofol forms a uniform fluid monolayer of high coverage and that propofol does not form islands at the interface.

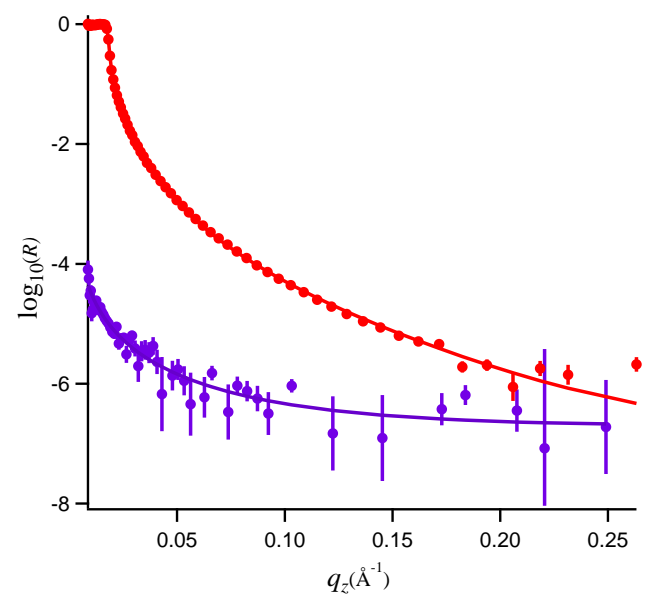

Figure 5. Neutron reflectometry data, $R$, and model fits for propofol solutions at the air/water interface recorded in $\mathrm{D}_{2} \mathrm{O}$ (red, top) and air contrast matched water (purple, bottom).

Propofol is considered to be of cylinder-like shape. The total volume of such cylinder, calculated from the inverse bulk density, is $285 \AA^{3}$. Close packing of such cylinder-like shapes accounts for the van der Waals volume of propofol ${ }^{56}$ which is $198 \AA^{3}, 11 \%$ water (from the NR analysis) and about $22 \%$ air of the total volume. Let's consider the two extreme cases. If the molecules (thought as cylinders) are considered to stand on their ends, and if the cylinder is considered to have a cross section area of $25 \AA^{2}$, slightly larger than for straight chain alkanes, then its length would be about $11 \AA$. The other extreme case would be a cylinder lying down giving an area per molecule of about $60 \AA^{2}$ and a thickness of about 5-6 A. Clearly, NR and tensiometry data are consistent with an intermediate situation (area per molecule 40-42 $\AA^{2}$, thickness $8.4 \AA$ ) , suggesting a tilted orientation of propofol with the OH-group towards the aqueous phase (the latter supported by the VSFS data discussed next). We are reluctant to go further and report a mean tilt angle as it would require a specific assumption about the packing of the molecules, which is not known a priori.

\section{VSF Spectra: $\mathrm{CH}$ region}

The VSF data in Figure 6a show well defined peaks, which testify that propofol adsorbs at the air/water interface in a non-random fashion. In Figure $6 \mathrm{~b}$ the attenuated total reflection (ATR)-IR spectrum of propofol in the $\mathrm{CH}$ region is presented, showing that the bulk IR peaks are reproduced in the surface VSF spectra. The assignments are based on published IR data $^{31,57}$ and VSF spectroscopy work on similar molecules. ${ }^{13,58-62}$ It is clear from Figure 6a that the SSP polarisation combination exhibits most of the vibrational features. The peak centred at $2873 \mathrm{~cm}^{-1}$ is assigned to the symmetric $\mathrm{CH}_{3}$ stretch $\left(\mathrm{sCH}_{3}\right)$, and is similar to the $\mathrm{sCH}_{3}$ peak found for alkyl chains. The small peak at $2910 \mathrm{~cm}^{-1}$, which is present only at high concentrations, is assigned to the $\mathrm{CH}$ of the isopropyl unit. The peak at $2965 \mathrm{~cm}^{-1}$ dominates the entire SSP spectrum and is assigned to the antisymmetric $\mathrm{CH}_{3}$ stretch $\left(\mathrm{aCH}_{3}\right)$. This peak is also observed in the PPP polarisation, but barely distinguishable in the SPS polarisation. There are two additional peaks in the SSP polarisation: the aromatic $\mathrm{CH}$ stretches at 3035 and $3071 \mathrm{~cm}^{-1}$, which are clearly distinguished at high concentrations.

For alkyl chains, the $\mathrm{aCH}_{3}$ peak is normally significantly weaker than the $\mathrm{sCH}_{3}$ peak in the SSP polarisation combination due to the chain orientation. ${ }^{63-65}$ However, for propofol the opposite is found. The reason is that the total VSF signal has contributions from all four closely spaced methyl groups (see Figure 1), and due to different directions of the transition dipole moments for the symmetric and antisymmetric methyl stretches, their signal strength will be differently enhanced. The same argument rationalises that the antisymmetric methyl stretch is stronger in SSP than PPP, which normally is not observed for alkyl chains. It should be pointed out that in different environments (e.g. without water as solvent) the propofol SSP spectrum (not shown) resembles a typical spectrum from an alkyl chain, which emphasises that the unusual SSP spectrum for propofol at the solution-air interface is due to the propofol orientation.

All VSF peaks discussed above are also present in the IR spectrum shown in Figure $6 \mathrm{~b}$. This includes the isopropyl $\mathrm{CH}$ peak at $2910 \mathrm{~cm}^{-1}$, which is observed as a small bump. Additionally, the IR spectrum shows the Fermi Resonance $\mathrm{CH}_{3}$ at $2935 \mathrm{~cm}^{-1}$ which is not clearly observed in the VSF spectra. The fitted amplitudes of the VSF peaks are provided in the Supporting Information. In Figure $6 \mathrm{c}$ the fitted amplitudes of the $\mathrm{sCH}_{3}$ and the $\mathrm{aCH}_{3}$ stretches are presented as a function of propofol concentration. Above $0.2 \mathrm{mM}$ the fitted amplitudes of both peaks approach a plateau, a result that is consistent with the 

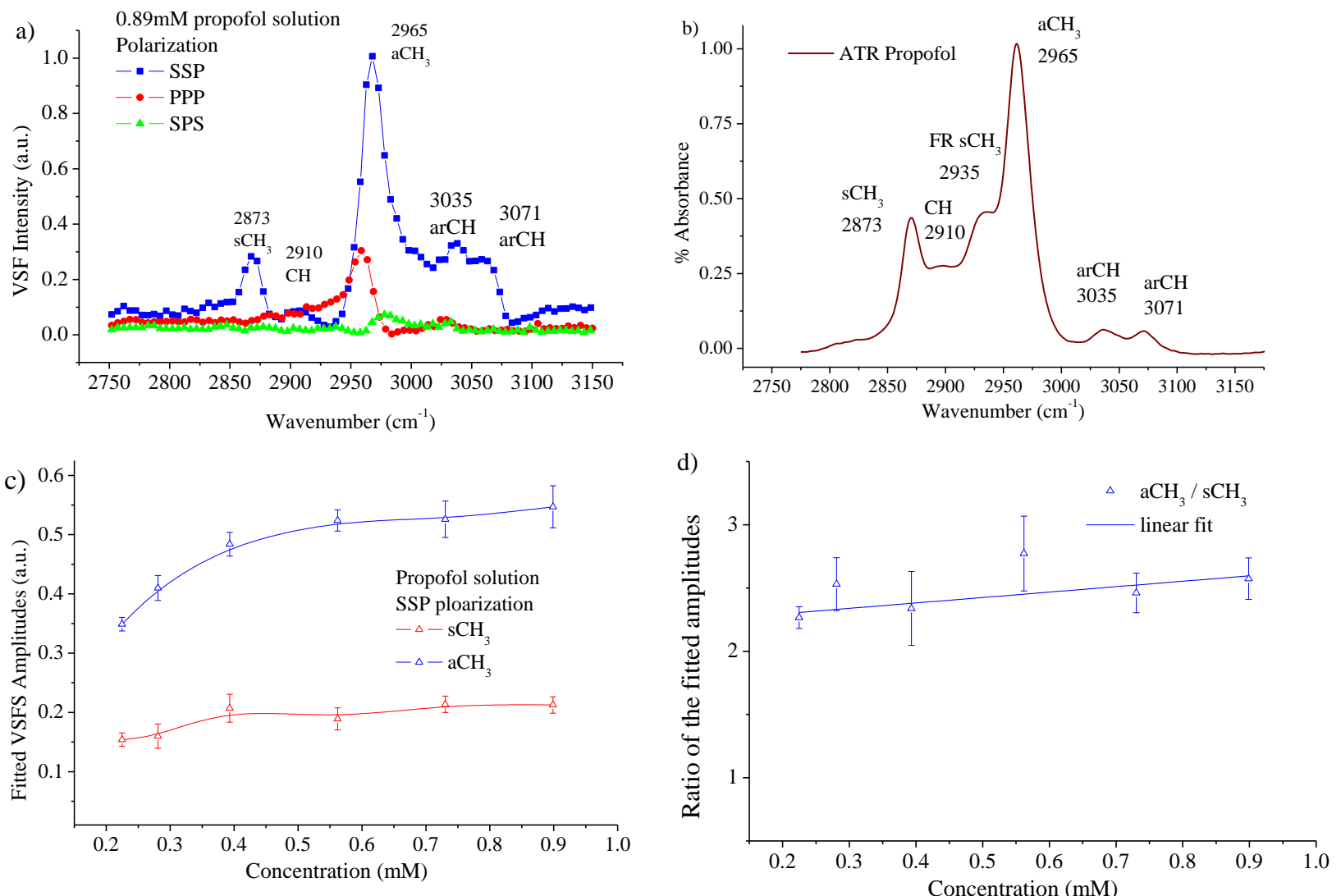

Figure 6. a) VSF spectra of a $0.89 \mathrm{mM}$ propofol solution in the SSP, PPP, and SPS polarisations. All peaks are normalised to the highest intensity peak - SSP aCH $\mathrm{H}_{3}$ b) ATR-IR spectrum of pure propofol in the $\mathrm{CH}$ stretching region; c) fitted amplitudes for the $\mathrm{SCH}_{3}$ stretch and the aCH $\mathrm{CH}_{3}$ stretch as a function of propofol concentration in the SSP polarisation. d) the ratio of the $\mathrm{aCH}_{3}$ to $\mathrm{SCH}_{3}$ amplitudes as a function of concentration. The line is a linear fit to the data.

findings from surface tension isotherms, as discussed earlier (Figure 3). The $\mathrm{sCH}_{3} / \mathrm{aCH} 3$ fitted amplitude ratio as a function of concentration is presented in Figure $6 \mathrm{~d}$. The fitted amplitude depends both on the average orientation and the number of molecules probed, whereas the $\mathrm{sCH}_{3} / \mathrm{aCH}_{3}$ ratio is only affected by the orientation, since the number density is cancelled by taking this ratio. We note that this ratio is almost constant over the concentration range $0.2-0.89 \mathrm{mM}$, meaning that the average orientation of the adsorbed propofol is almost constant. The SPS polarisation did not show any $\mathrm{CH}$ feature that could be used reliably in the fitting procedure. This, and the fact that there are four methyl groups pointing in different directions that contribute to that signal, prohibits a more detailed orientational analysis. The assignments of vibrational features of propofol in the $\mathrm{CH}$ stretching region are presented in Table 1.

\section{VSF Spectra: Water Region}

In order to have a reference between different measurement sessions, spectra of the free $\mathrm{OH}$ vibration of the pure water surface were recorded before all measurements. In Figure 7 spectra of a 0.89 $\mathrm{mM}$ propofol solution together with the pure water spectra in the SSP and PPP polarisations are shown.

Both the SSP and the PPP spectra of water agree with the spectra recorded before, ${ }^{17,63,68}$ where the most prominent features exhibited in the SSP polarisation are the broad band spanning over several hundred wavenumbers and centred at around 3300 $\mathrm{cm}^{-1}$ as well as the sharp peak at $3704 \mathrm{~cm}^{-1}$.

The latter peak, which is present in both the SSP and PPP polarisation, is assigned to the $\mathrm{OH}$ stretching mode of the topmost surface $\mathrm{OH}$ bonds of water molecules protruding into air and vibrating free from hydrogen bonding. ${ }^{69}$ The assignment of the broad band was for a long time a source of debate. ${ }^{70-72}$ However, it is broadly agreed now that the broad band from 3150 to $3450 \mathrm{~cm}^{-1}$ is assigned to hydrogen bonded water molecules with varying strength and coordination meaning that the $\mathrm{OH}$ signal is a signature of a collective vibration of several water molecules. ${ }^{73-}$ 
Table 1. Assignments of vibrational features of propofol in the $\mathrm{CH}$ stretching region.

\begin{tabular}{|l|l|l|l|l|}
\hline Peak $\left(\mathrm{cm}^{-1}\right)$ & Polarisation & Observed & Assignments & Refs. \\
\hline 2873 & SSP & VSF/IR & $\mathrm{SCH}_{3}$ & $13,57,66$ \\
\hline 2910 & SSP, PPP & VSF/IR & $\mathrm{CH}$ isopropyl & 67 \\
\hline 2965 & SSP, PPP, SPS & VSF/IR & $\mathrm{aCH}_{3}$ & $13,57,61,62$ \\
\hline 3035 & SSP, PPP & VSF/IR & $\operatorname{arCH}$ & 13,57 \\
\hline 3071 & SSP & VSF/IR & $\operatorname{arCH}$ & 13,57 \\
\hline
\end{tabular}

The SSP polarisation spectrum of a $0.89 \mathrm{mM}$ propofol solution, shown in Figure $7 \mathrm{a}$, is considerably different from the pure water spectrum. At this propofol concentration, the free $\mathrm{OH}$ peak at $3704 \mathrm{~cm}^{-1}$ has essentially vanished, suggesting that none or very few free $\mathrm{OH}$ bonds remain. However, a new broad band, centred around $3670 \mathrm{~cm}^{-1}$, appears. A similar band has been observed for several amphiphilic molecules (e.g., decanol, sugar surfactants and alkyl polyethylene glycol surfactants) at the air/water interface, and has been assigned to the $\mathrm{OH}$ stretch of ordered water molecules weakly interacting with hydrocarbon moieties. ${ }^{17}$ This is consistent with the relatively large hydrophobic part of propofol, and suggests direct contact between water and this region of propofol. The small red shift of around $30 \mathrm{~cm}^{-1}$ compared to the free $\mathrm{OH}$ vibration indicates that the interaction is weak. We note that since propofol is non-ionic, any significant enhancement of the water signal as observed for ionic amphiphiles ${ }^{77,78}$ is neither expected nor seen.

In the PPP spectrum in Figure $7 \mathrm{~b}$ a band covering nearly the whole $\mathrm{OH}$ stretching region is observed, with the maximum intensity around 3600 $\mathrm{cm}^{-1}$. Thus, the maximum is red shifted with around 70 $\mathrm{cm}^{-1}$ in comparison with the SSP spectrum, which exhibits nearly zero intensity at $3600 \mathrm{~cm}^{-1}$. The $\mathrm{OH}$ bond populations responsible for the peaks at 3600 $\mathrm{cm}^{-1}$ and $3670 \mathrm{~cm}^{-1}$ possess weak interactions, and are thus excluded from hydrogen bonding due to proximity to the hydrophobic moieties of propofol. However, since the two peaks have their maximum at different wavenumbers, the two populations experience dissimilar environments.

An interesting observation is that the right hand side of the broad band, towards $3400 \mathrm{~cm}^{-1}$ is almost completely suppressed in the SSP polarisation combination (Figure 7a). Instead, a new band centred at about $3175 \mathrm{~cm}^{-1}$ clearly shows up in the spectrum. The fact that this band appears at such low frequency, close to the centre frequency for ice, ${ }^{79,80}$ suggests that it is associated with interfacial water that experience strong interactions with the hydroxyl part of propofol, similar to what was found for long chain alcohols. ${ }^{20} \mathrm{~A}$ similar band, centred at $3150 \mathrm{~cm}^{-1}$ has further been observed for interfacial sugar surfactants possessing $\mathrm{OH}$ groups (C10 maltoside and $\mathrm{C} 10$ glucoside) and assigned to $\mathrm{OH}$ stretching vibrations of the hydroxyl groups in the sugar rings and their hydration shells. ${ }^{17}$ In contrast, 1-decanol, which, like propofol, contains a single $-\mathrm{OH}$ group and a large hydrophobic moiety, exhibited a band centred at $3250 \mathrm{~cm}^{-1}$, thus indicating relatively weaker hydrogen bonds.
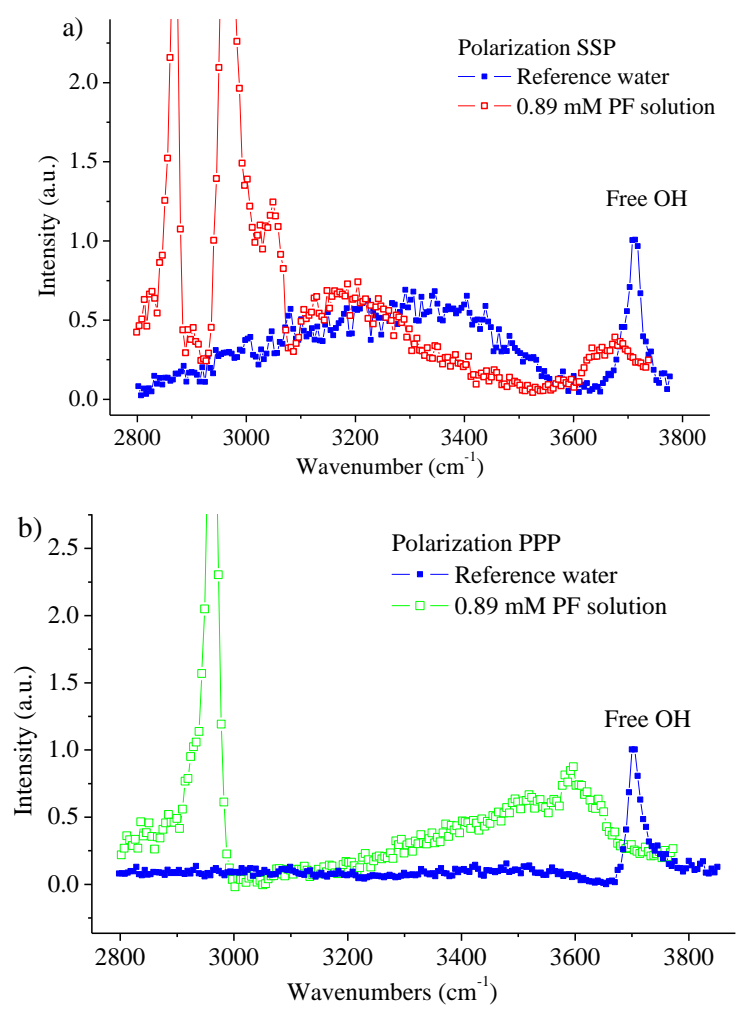

Figure 7. Water region spectra of a $0.89 \mathrm{mM}$ propofol solution together with a pure water spectrum in a) SSP, and b) PPP polarisation combination. These two spectra are normalised to the intensity of the free $\mathrm{OH}$ vibration at the pure water surface. 
Moreover, the PPP spectrum of 1-decanol showed a broad band extending over the region 3000 - $3800 \mathrm{~cm}-1$, obviously different from that obtained in presence of propofol. Accordingly, the strength of the hydrogen bonds of water that are hydrating propofol (SSP spectra) is in between those observed with the sugar surfactants and decanol, which indicates that the hydrogen bond strength depends not only on the hydrophilic group, which is the same for decanol and propofol, but also the surrounding hydrophobic environment. $^{20}$

The freedom of the molecules to rotate or move around may also influence the strength of the hydrogen bond. Propofol has a larger area per molecule then decanol ${ }^{17}$ and therefore has less constraints in adopting a position that allows for stronger hydrogen bonds to be formed.

\section{Summary and Conclusion}

Propofol does not form dimers or multimers in bulk solution up to the solubility limit $(0.89 \mathrm{mM})$. It adsorbs at the air/water interface forming a dense (volume fraction 0.86) uniform film (area/molecule $\approx$ $40-42 \AA^{2}$, thickness $\approx 8.4 \AA$ ) close to the solubility limit. The propofol molecule is tilted relative the surface normal and oriented with the $\mathrm{OH}$-group towards water. Its orientation at the interface is almost constant in the concentration range $0.2-0.89 \mathrm{mM}$.

We have identified different water populations that hydrate propofol at the air/aqueous solution interface. Strong hydrogen bonds, similar to those found for the tetrahedrally-coordinated hydrogen bonding in ice, is formed between water and the $\mathrm{OH}$-group of propofol. These hydrogen bonds are stronger than those found between water and decanol, but weaker than those found next to sugar surfactants. Thus, the hydrogen bond strength does not only depend on the hydrophilic group, which is the same for decanol and propofol, but also on the surrounding hydrophobic environment and the ability to adopt conformations that allow formation of strong hydrogen bonds.

Effective molecular information regarding the arrangement and hydration of propofol at the air/water interface opens the door for a more extensive examination of the interfacial properties of propofol, especially at the buried membrane/water interfaces where this may have important implications for understanding the driving forces for the underlying interactions of drugs in model systems, and they set the stage for us and others to proceed with studies of the interactions of propofol and other small model drugs with more complex interfacial morphologies.

\section{Author Information}

Corresponding Authors:

Email: petru.niga@ri.se and magnusj@kth.se

\section{Conflicts of interest}

There are no conflicts to declare

\section{Acknowledgements.}

This work was kindly supported by Omya International AG. We thank the Institut Laue-Langevin for an allocation of beam time on FIGARO (DOI: 10.5291/ILLDATA.TEST-2589) as well as access to complementary instruments in the Partnership for Soft Condensed Matter. JD and PC thank the NanoS3-290251 ITN and the Swedish Research Council VR for support.

\section{References:}

1. J. C. D. F. Franks, J. L. Finney, J. E. Quinn, J. O. Baum, F. Franks, J. E. Desnoyers, Water Science Reviews, 2009.

2. F. Franks and Editor, Water: A Comprehensive Treatise: The Physics and Physical Chemistry of Water, Plenum, New York, 1972.

3. A. P. G. Arthur W. Adamson, Physical Chemistry of Surfaces, John Wiley \& Sons, Inc. , 1997.

4. J. Grimshaw, in Electrochemical Reactions and Mechanisms in Organic Chemistry, ed. J. Grimshaw, Elsevier Science B.V., Amsterdam, 2000, pp. 261-299.

5. M. Patra, E. Salonen, E. Terama, I. Vattulainen, R. Faller, B. W. Lee, J. Holopainen and M. Karttunen, Biophysical Journal, 2006, 90, 11211135.

6. A. R. Mazzeo, J. Nandi and R. A. Levine, American Journal of Physiology Gastrointestinal and Liver Physiology, 1988, 254, G57-G64.

7. G. Banfalvi, in Permeability of Biological Membranes, Springer International Publishing, Cham, 2016, DOI: 10.1007/978-3-319-280981_1, pp. 1-71.

8. A. K. Lugli, C. S. Yost and C. H. Kindler, European journal of anaesthesiology, 2009, 26, 807-820. 
9. F. H. Frimmel, Angewandte Chemie, 1993, 105, 800-800.

10. J. W. Whalen, Journal of Chemical Education, 1983, 60, A322.

11. C. M. Johnson and S. Baldelli, Chemical Reviews, 2014, 114, 8416-8446.

12. J. Sung, K. Park and D. Kim, The Journal of Physical Chemistry B, 2005, 109, 18507-18514.

13. R. Lu, W. Gan, B.-h. Wu, Z. Zhang, Y. Guo and H.-f. Wang, The Journal of Physical Chemistry $B, 2005,109,14118-14129$.

14. G. Ma and H. C. Allen, The Journal of Physical Chemistry B, 2003, 107, 6343-6349.

15. Y. Rao, M. Subir, E. A. McArthur, N. J. Turro and K. B. Eisenthal, Chemical Physics Letters, 2009, 477, 241-244.

16. W.-T. Liu, L. Zhang and Y. R. Shen, The Journal of Chemical Physics, 2006, 125, 144711.

17. E. Tyrode, C. M. Johnson, A. Kumpulainen, M. W. Rutland and P. M. Claesson, Journal of the American Chemical Society, 2005, 127, 1684816859.

18. S. Baldelli, C. Schnitzer, M. J. Shultz and D. J. Campbell, The Journal of Physical Chemistry B, 1997, 101, 4607-4612.

19. Y.-C. Wen, S. Zha, C. Tian and Y. R. Shen, The Journal of Physical Chemistry C, 2016, 120, 15224-15229.

20. J. A. Mondal, V. Namboodiri, P. Mathi and A. K. Singh, The Journal of Physical Chemistry Letters, 2017, 8, 1637-1644.

21. C. D. Stanners, Q. Du, R. P. Chin, P. Cremer, G. A. Somorjai and Y. R. Shen, Chemical Physics Letters, 1995, 232, 407-413.

22. V. B. Fainerman, R. Miller and H. Möhwald, The Journal of Physical Chemistry B, 2002, 106, 809-819.

23. J. A. Killian and G. von Heijne, Trends in Biochemical Sciences, 2000, 25, 429-434.

24. A. F. Miller, R. W. Richards and J. R. P. Webster, Macromolecules, 2000, 33, 76187628.

25. D. M. Small, L. Wang and M. A. Mitsche, Journal of Lipid Research, 2009, 50, S329-S334.

26. L. Du, X. Liu, W. Huang and E. Wang, Electrochimica Acta, 2006, 51, 5754-5760.

27. R. J. Alsop, C. L. Armstrong, A. Maqbool, L. Toppozini, H. Dies and M. C. Rheinstadter, Soft Matter, 2015, 11, 4756-4767.
28. Y. Saaka, D. T. Allen, Y. Luangwitchajaroen, Y. Shao, R. A. Campbell, C. D. Lorenz and M. J. Lawrence, Soft Matter, 2018, 14, 3135-3150.

29. P. Niga, P. M. Hansson-Mille, A. Swerin, P. M. Claesson, J. Schoelkopf, P. A. C. Gane, E. Bergendal, A. Tummino, R. A. Campbell and C. M. Johnson, J Colloid Interface Sci, 2018, 526, 230-243.

30. I. Vasileiou, T. Xanthos, E. Koudouna, D. Perrea, C. Klonaris, A. Katsargyris and L. Papadimitriou, European Journal of Pharmacology, 2009, 605, 1-8.

31. A. Mohammad, F. B. Faruqi and J. Mustafa, Journal of Cancer Therapy, 2010, 1, 124-130.

32. R. A. Siddiqui, M. Zerouga, M. Wu, A. Castillo, K. Harvey, G. P. Zaloga and W. Stillwell, Breast Cancer Research, 2005, 7, R645-R654.

33. G. Fragneto, The European Physical Journal Special Topics, 2012, 213, 327-342.

34. Y. Gerelli, L. Porcar and G. Fragneto, Langmuir, 2012, 28, 15922-15928.

35. T. Soranzo, D. K. Martin, J.-L. Lenormand and E. B. Watkins, Scientific Reports, 2017, 7, 3399.

36. S. Isaksson, E. B. Watkins, K. L. Browning, T. Kjellerup Lind, M. Cárdenas, K. Hedfalk, F. Höök and M. Andersson, Nano Letters, 2017, 17, 476-485.

37. R. A. Campbell, H. P. Wacklin, I. Sutton, R. Cubitt and G. Fragneto, The European Physical Journal Plus, 2011, 126, 107.

38. J. R. Lu, R. K. Thomas and J. Penfold, Advances in Colloid and Interface Science, 2000, 84, 143304.

39. J. Hernandez-Pascacio, Á. Piñeiro, J. M. Ruso, N. Hassan, R. A. Campbell, J. Campos-Terán and M. Costas, Langmuir, 2016, 32, 66826690.

40. Á. Ábraham, R. A. Campbell and I. Varga, Langmuir, 2013, 29, 11554-11559.

41. R. A. Campbell, A. Tummino, B. A. Noskov and I. Varga, Soft Matter, 2016, 12, 5304-5312.

42. R. A. Campbell, Y. Saaka, Y. Shao, Y. Gerelli, R. Cubitt, E. Nazaruk, D. Matyszewska and M. J. Lawrence, Journal of Colloid and Interface Science, 2018, 531, 98-108.

43. A. Nelson, Journal of Applied Crystallography, 2006, 39, 273-276.

44. P. Niga, PhD, Royal Institute of Technology, 2010. 
45. X. Chen, M. L. Clarke, J. Wang and Z. Chen, International Journal of Modern Physics B: Condensed Matter Physics, Statistical Physics, Applied Physics, 2005, 19, 691-713.

46. M. Buck and M. Himmelhaus, Journal of Vacuum Science \& Technology, A: Vacuum, Surfaces, and Films, 2001, 19, 2717-2736.

47. M. J. Shultz, C. Schnitzer, D. Simonelli and S. Baldelli, International Reviews in Physical Chemistry, 2000, 19, 123-153.

48. X. Zhuang, P. B. Miranda, D. Kim and Y. R. Shen, Physical Review B: Condensed Matter and Materials Physics, 1999, 59, 12632-12640.

49. X. D. Zhu, H. Suhr and Y. R. Shen, Physical Review B: Condensed Matter and Materials Physics, 1987, 35, 3047-3050.

50. C. Hirose, N. Akamatsu and K. Domen, Applied Spectroscopy, 1992, 46, 1051-1072.

51. L. F. G. Reiner, E. Paula, M. Perillo and D. García,, Journal of Biomaterials and Nanobiotechnology, 2013, 4, 28-34.

52. K. I. Momot, P. W. Kuchel, B. E. Chapman, P. Deo and D. Whittaker, Langmuir, 2003, 19, 2088-2095.

53. L. Fielding, Tetrahedron, 2000, 56, 6151-6170.

54. J. Alsins, M. Björling, I. Furó and V. Egle, Journal of Physical Organic Chemistry, 1999, $12,171-175$.

55. I. León, J. Millán, E. J. Cocinero, A. Lesarri and J. A. Fernández, Angewandte Chemie International Edition, 2014, 53, 12480-12483.

56. K. A. Woll, B. P. Weiser, Q. Liang, T. Meng, A. McKinstry-Wu, B. Pinch, W. P. Dailey, W. D. Gao, M. Covarrubias and R. G. Eckenhoff, ACS Chemical Neuroscience, 2015, 6, 927-935.

57. A. Jabłońska, Ł. Ponikiewski, K. Ejsmont, A. Herman and A. Dołęga, Journal of Molecular Structure, 2013, 1054-1055, 359-366.

58. P. Guyot-Sionnest, R. Superfine, J. H. Hunt and Y. R. Shen, Chemical Physics Letters, 1988, 144, 1-5.

59. C.-y. Wang, H. Groenzin and M. J. Shultz, Journal of the American Chemical Society, 2005, 127, 9736-9744.

60. T. Ishihara, T. Ishiyama and A. Morita, The Journal of Physical Chemistry C, 2015, 119, 9879-9889.

61. C.-y. Wang, H. Groenzin and M. J. Shultz, The Journal of Physical Chemistry B, 2004, 108, 265-272.
62. Y. Yu, Y. Wang, K. Lin, N. Hu, X. Zhou and S. Liu, The Journal of Physical Chemistry A, 2013, 117, 4377-4384.

63. P. Niga, Self Assembly at the Liquid Air Interface, PhD Thesis, Stockholm 2010.

64. J. F. D. Liljeblad, V. Bulone, E. Tyrode, M. W. Rutland and C. M. Johnson, Biophys. J. Letters, 2010, 98, L50-L52.

65. E. Tyrode, P. Niga, M. Johnson and M. W. Rutland, Langmuir, 2010, 26, 14024-14031.

66. H. Chen, W. Gan, R. Lu, Y. Guo and H.-F. Wang, Journal of Physical Chemistry B, 2005, 109, 8064-8075.

67. S. Kataoka and P. S. Cremer, Journal of the American Chemical Society, 2006, 128, 55165522.

68. E. Tyrode, C. M. Johnson, M. W. Rutland and P. M. Claesson, Journal of Physical Chemistry C, 2007, 111, 11642-11652.

69. Q. Du, R. Superfine, E. Freysz and Y. R. Shen, Physical Review Letters, 1993, 70, 2313-2316.

70. G. L. Richmond, Annu. Rev. Phys. Chem., 2001, 52, 357-389.

71. D. F. Liu, G. Ma, L. M. Levering and H. C. Allen, Journal Of Physical Chemistry B, 2004, 108, 2252-2260.

72. M. Sovago, R. K. Campen, G. W. H. Wurpel, M. Müller, H. J. Bakker and M. Bonn, Physical Review Letters, 2008, 100, 173901.

73. T. Ishiyama and A. Morita, J. Phys. Chem. A, 2007, 111, 9277-9285.

74. A. Morita and J. T. Hynes, Chemical Physics, 2000, 258, 371-390.

75. S. Yamaguchi, The Journal of Chemical Physics, 2015, 143, 034202.

76. A. Adhikari, The Journal of Chemical Physics, 2015, 143, 124707.

77. M. R. Watry, T. L. Tarbuck and G. L. Richmond, Journal of Physical Chemistry B, 2003, 107, 512-518.

78. X. Chen, W. Hua, Z. Huang and H. C. Allen, Journal of the American Chemical Society, 2010, 132, 11336-11342.

79. R. Claverie, M. D. Fontana, I. Duričković, P. Bourson, M. Marchetti and J.-M. Chassot, Sensors, 2010, 10, 3815.

80. Q. Sun and H. Zheng, Progress in Natural Science, 2009, 19, 1651-1654. 